\title{
Sex Differences in Causes of Death After Stroke: Evidence from a National, Prospective Registry
}

\author{
Hoang T. Phan, PhD, ${ }^{13}$ Seana Gall, PhD, ${ }^{1}$ Christopher L. Blizzard, PhD, ${ }^{1}$ Natasha A. Lannin, $\mathrm{PhD}, 4,5$ \\ Amanda G. Thrift, PhD, ${ }^{3}$ Craig S. Anderson, PhD, ${ }^{6}$ Joosup Kim, PhD, ${ }^{3,7}$ Rohan Grimley, FRACP, ${ }^{3,8}$ \\ Helen C. Castley, FRACP, ${ }^{9}$ Monique F. Kilkenny, PhD, ${ }^{3,7}$ Dominique A. Cadilhac, PhD, ${ }^{3,7}$ \\ on Behalf of the Australian Stroke Clinical Registry Consortium
}

\begin{abstract}
Background: We examined sex differences in cause of death and cause-specific excess mortality after stroke. Materials and Methods: First-ever strokes (2010-2013; 35 hospitals) participating in the Australian Stroke Clinical Registry were linked to national death registrations and other administrative datasets. One-year causespecific mortality was categorized as stroke, ischemic heart disease, other cardiovascular disease (CVD; e.g., hypertension), cancer, and other. Specific hazard ratios (sHRs) of death for women compared to men were estimated using competing risk models, with adjustment for factors differing by sex (e.g., age and stroke severity). Age- and sex-specific mortality rates expected in the general population were derived from national data. Standardized mortality ratios (SMRs; observed/expected deaths) were estimated for cause-specific mortality by sex after age standardization.

Results: Among 9,441 cases (46\% women), women were 7 years older than men, had more severe strokes, and received similar patterns of suboptimal secondary prevention medications at discharge. Women had greater mortality associated with stroke ( $\left.\mathrm{sHR}_{\text {unadjusted }} 1.65\right)$ and other CVD ( $\left.\mathrm{sHR}_{\text {unadjusted }} 1.65\right)$, which was related to age and stroke severity rather than other factors. Compared to population norms, those surviving to 30 days had eight-fold increased mortality from stroke (primary/recurrent) events irrespective of sex $\left(\mathrm{SMR}_{\text {age-standardised }}\right.$ women 8.8; men 8.3). Excess mortality from other CVD was greater in women $\left(\mathrm{SMR}_{\text {age-standardised }} 3.6 \mathrm{vs.}\right.$ men $2.8 ; p \quad 0.026)$.

Conclusions: Cause-specific mortality after first-ever stroke differs by sex. The greater death rate attributed to stroke/other CVD in women was mostly explained by age and stroke severity. Greater implementation of secondary stroke prevention is relevant to both sexes.
\end{abstract}

Keywords: stroke, cause of death, sex difference, care, excess mortality

\section{Introduction}

W OMEN HAVE BEEN reported to have greater all-cause mortality in the long term after stroke than men. ${ }^{1,2} \mathrm{We}$ have previously reported that the sex difference in 1- and 5 -year mortality is mostly associated with deaths in the first
6 months. ${ }^{1}$ It may be that there are sex differences in cause of death after stroke that potentially vary over time. In the studies of cause-specific mortality up to 10 years after stroke, sexaggregated data are generally lacking, ${ }^{3-6}$ and sex has been used as a variable for adjustment in multivariable models. ${ }^{4,5}$ Earlier studies had incomplete use of neuroimaging and other

\footnotetext{
${ }^{1}$ Menzies Institute for Medical Research, University of Tasmania, Hobart, Australia.

${ }^{2}$ Department of Public Health Management, Pham Ngoc Thach University of Medicine, Hồ Chí Minh, Vietnam.

${ }^{3}$ Department of Medicine, School of Clinical Sciences at Monash Health, Monash University, Melbourne, Australia.

${ }^{4}$ Department of Neuroscience, Central Clinical School, Monash University, Melbourne, Australia.

${ }^{5}$ Alfred Health, Melbourne, Australia.

${ }^{6}$ Faculty of Medicine, The George Institute for Global Health, The University of New South Wales, Sydney, Australia.

${ }^{7}$ Stroke Theme, The Florey Institute of Neuroscience and Mental Health, Heidelberg, Victoria, Australia.

${ }^{8}$ Sunshine Coast Clinical School, University of Queensland, Birtinya, Australia.

${ }^{9}$ Neurology Department, Royal Hobart Hospital, Hobart, Australia.
} 
investigations that compromised reliability of diagnoses, ${ }^{4,5}$ Furthermore, there is some evidence that women receive evidence-based care less often than men. ${ }^{7,8}$ It is uncertain whether such differences in stroke care contribute to the sex differences in cause-specific mortality following stroke. Differences in the attributable causes for excess mortality after stroke compared to the general population, including any difference by sex, are also limited and data are outdated. ${ }^{6}$

The aims of this study were to (i) examine whether the cause-specific mortality up to 1 year after stroke varies by sex, and over time, among hospitalized people with acute stroke in Australia, (ii) identify the factors, including access to evidence-based care, which may account for any sex difference in cause-specific mortality, and (iii) calculate the excess mortality attributable to stroke and other causes compared to the general population.

\section{Materials and Methods}

\section{Study design}

Data are from the Australian Stroke Clinical Registry (AuSCR), a nationwide, multicenter, prospective registry that incorporates standardized methods of data collection using a validated system to assess the quality of acute stroke care and outcomes in Australia., ${ }^{9,10}$

\section{Institutional review board statement}

This study was approved by the Tasmanian Health and Medical Human Research Ethics Committee (H0015287); the AuSCR Research Task Group and Management Committee (08/2016); and appropriate ethics committees and/or governance bodies at all participating hospitals and the Australian Institute of Health and Welfare (AIHW) for data linkage to national death registrations, known as the National Death Index (NDI). Participants are made aware that their data may be used for secondary research purposes which is outlined in the ethics approved patient information form for AuSCR. Due to the lag times for receiving cause-specific mortality data from the NDI, ${ }^{11}$ our study was limited to cases of first-ever stroke registered in the AuSCR at 35 hospitals during 20102013.

\section{Variables}

Covariates used in the analyses included patient characteristics and key in-hospital care processes that might explain the sex differences in cause-specific after stroke. These covariates were categorized into five groups, including: (i) sociodemographics, (ii) behavioral risk factors and comorbidities, (iii) stroke-related factors (e.g., stroke severity, stroke type, and known pathological cause of ischemic stroke defined according to the TOAST criteria), ${ }^{12}$ (iv) processes of care provided in the hospital, including four national indicators (access to stroke unit care, intravenous thrombolysis if an ischemic stroke (IS), care plan provided at discharge, and being discharged on antihypertensive agents), and four additional indicators collected in the state of Queensland (aspirin administration $\leq 48$ hours if an IS, mobilization during admission, swallow screen, and being discharged on antiplatelets or antithrombotic medications (IS), and (v) discharge information (length of hospital stay and discharge destination), and 90180 day factors (living arrangements, self-reported recurrent stroke event, and hospital readmission; see Supplementary Data S1).
Through the Stroke123 data linkage project, ${ }^{13}$ information on risk factors for stroke and comorbidities was obtained from administrative hospital records. These were based on the International Statistical Classification of Diseases and Related Health Problems 10th Revision (ICD-10) codes using a "look-back period" of 5 years before and including the stroke event. The specific comorbidities were used to provide a Charlson Cormorbidity Index score for each patient. ${ }^{14}$

The ability to walk independently on admission was used as a proxy assessment of stroke severity. ${ }^{1,15}$ Stroke severity, indicated by the walking ability on admission, has been established as a predictor of all-cause mortality and contributing factor to the greater 1-year mortality in women following stroke using the same dataset. ${ }^{2}$ We hypothesized that stroke severity would also be associated with specific-cause mortality following stroke, and examined the role of stroke severity on the association between sex and individual cause of death.

\section{Outcomes}

All-cause and cause-specific mortality up to 1 year after stroke were obtained by linkage of patient-level NDI data through the AIHW, where all deaths are certified by a medical practitioner on a Medical Certificate of Cause of Death or by a coroner. ${ }^{11}$ The data in ICD-10 codes underwent quality checks by trained staff. ${ }^{11}$ Primary causes of death were classified into stroke, ischemic heart disease (IHD), other cardiovascular disease (CVD; e.g., hypertension, atrial fibrillation, heart failure, peripheral vascular diseases, and others), cancer, other causes (including other diseases; accidents [e.g., falls]; medical complications; and unclassified conditions), and unknown causes (missing ICD-10 codes on death records).

\section{Statistical analysis}

Multilevel competing risk modeling, accounting for hospital as a unit of patient clusters, was used to estimate specific hazard ratios (sHRs) of specific cause of death for women compared to men. Estimates of sex differences in processes of care and mortality were performed for all hospitals and in relevant subgroups (see Statistical Analysis section in Supplementary Data S1).To assess the role of covariates on the association between sex and outcomes, we used purposeful model building. ${ }^{16}$ Variables were entered into the model only if they met all three criteria that defined them as potential confounding factors $^{16}$ : (i) the covariate was associated with the outcomes, $p<0.1$; (ii) the covariate was associated with sex, $p<0.1$; and (iii) the inclusion of the covariate changed the magnitude of the coefficient for the sex difference in the outcome ([unadjusted $\beta$ - adjusted $\beta$ ]/unadjusted $\beta \times 100 \%$ ) by $\geq 10 \%$. The modelbuilding process was initially undertaken for each covariate separately, and then confounding or contributing factors were included in the multivariable models. To minimize the impact of missing data for multivariable models, those with missing age and stroke severity were excluded (6\%). The modifying effect of time to death and other covariates on sex differences was assessed by a test of the statistical significance of a coefficient of a sex $\times$ covariate product term.

Comparison of patients with stroke and the general population. Cause-specific excess mortality up to 1 year after stroke was assessed for stroke, IHD, other CVD (e.g., hypertension), and cancer. Age- and sex-specific mortality 
rates for each cause of death by calendar year expected in the general population were derived from Australian data (stroke and other CVD: Australian Heart Disease Statistics 2016 by the National Heart Foundation ${ }^{17}$ and cancer: data published by $\mathrm{AIHW}^{18}$ ). Expected deaths were estimated for each sex by multiplying the age-specific person-years of observation in the study group (stroke registrants) by the death rate of the general population; values were summed for each age group to calculate the number of expected deaths. We then estimated the standardized mortality ratio (SMR) as the quotient of observed to expected numbers of deaths for each cause, separately for women and men. Absolute excess risk (AER) for each cause of death was also estimated to compare the absolute additional mortality between men and women at 1 year after stroke $($ AER $=[$ expected - observed deaths $] /$ agespecific person-years at risk $\times 100$ ). As stroke-specific mortality is largely driven by deaths in the first 30 days after onset, we reported the 1-year SMR and 1-AER in 30-day survivors (often called nonfatal strokes ${ }^{6}$ ). Data are reported with $95 \%$ confidence intervals (CIs) and a two-tailed $p$-value $\leq 0.05$ was considered statistically significant. All analyses were performed using Stata 12.1 (StataCorp., TX). ${ }^{19}$

\section{Results}

There were 9,441 patients (median age 75 years [interquartile range 6484 ]; $46.4 \%$ female) with first-ever stroke (81\% ischemic) during 20102013. Based on sociodemographic and stroke-related characteristics (Table 1), women were $\sim 7$ years older than men and $9.3 \%$ fewer women than men could walk independently upon admission. The median Charlson Comorbidity Index scores in 8,819 patients ( $>93 \%$ matches with administrative data) were similar between men and women (Table 1). However, there were sex differences in some individual risk factors or comorbidities with more women having dementia, hypertension, atrial fibrillation, and heart failure/myocardial infarction, while more men were smokers and had hyperlipidemia and diabetes mellitus. Women had fewer known causes of stroke and experienced more stroke events, while in hospital for another condition (inhospital stroke) than men (Supplementary Table S1).

\section{Sex differences in care and outcomes}

We found that men and women generally received similar acute care (Table 2 ). The exception was that $\sim 10.6 \%$ fewer women were administered aspirin $\leq 48$ hours than men based on the subset of patients from Queensland ( $n=21$ hospitals; $n=2,972$ ), regardless of whether or not early deaths $\leq 48$ hours were excluded.

When compared to men, women were 2.2 times more often discharged to aged care facility, 1.3 times less often discharged directly home from acute care, and $\sim 50 \%$ more likely to live alone at 90180 days at stroke (Supplementary Table S1).

\section{Sex difference in cause-specific mortality after stroke}

Women had greater all-cause mortality ( $25.4 \%$ vs. men $19.1 \% ; p<0.001)$ at 1 year after a first-ever stroke. Stroke and CVD (i.e., IHD and other cardiovascular conditions) accounted for over half of all deaths within 1 year following stroke. However, the distribution of cause of death differed between the sexes. Among those deceased by any cause $(n=2,080$; Fig. 1 and Supplementary Table S2), men had more deaths due to cancer (12\% vs. women 6\%) and IHD (8\% vs. women $6 \%$ ), while women had more deaths attributed to stroke (50\% vs. men $41 \%)$ or other CVD (16\% vs. men $13 \%)$. More specific details of each cause are provided in Supplementary Table S2. The proportion of missing ICD codes for cause of death was comparable between the sexes (women $9 \%$ and men $10 \%$ ). Further details of the distribution of cause of death for women and men by age group and time to death are provided in the Supplementary Figures S1 and S2.

Table 1. Characteristic of Australian Stroke Clinical Registry Registrants for First-Ever Stroke During 2010-2013 ( $N=35$ Hospitals) By SeX

\begin{tabular}{|c|c|c|c|}
\hline & $\operatorname{Men} \mathrm{n}(\%)^{\mathrm{a}}$ & Women $\mathrm{n}(\%)^{\mathrm{a}}$ & $\mathrm{p}$-Value \\
\hline Number of cases & $5,059(53.6)$ & $4,382(46.4)$ & $<0.001$ \\
\hline Age, median (IQR) $)^{\mathrm{b}}$ & $72.0(61.8-81.0)$ & $78.8(67.8-85.9)$ & $<0.001$ \\
\hline Walking independently at admission (proxy for less severe stroke) ${ }^{b}$ & $1,949(40.9)$ & 1,304 (31.6) & $<0.001$ \\
\hline \multicolumn{4}{|l|}{ Comorbidities $^{\mathrm{c}}(N=8,819)$} \\
\hline Number of cases & $4,716(53.5)$ & $4,103(46.5)$ & $<0.001$ \\
\hline Charlson Comorbidity Index score, median (min $\max$ ) & $3\left(\begin{array}{ll}0 & 12\end{array}\right)$ & $3\left(\begin{array}{ll}0 & 12\end{array}\right)$ & 0.192 \\
\hline \multicolumn{4}{|l|}{ Individual comorbidities } \\
\hline Dementia & $226(4.8)$ & $317(7.7)$ & $<0.001$ \\
\hline Hypertension & $3,309(70.2)$ & $3,050(74.4)$ & $<0.001$ \\
\hline Smoking & $1,179(25.0)$ & $658(16.0)$ & $<0.001$ \\
\hline Hyperlipidemia & $710(15.1)$ & $521(12.7)$ & 0.001 \\
\hline Atrial fibrillation & $1,362(28.9)$ & $1,457(35.5)$ & $<0.001$ \\
\hline Heart failure/myocardial infarction & $910(19.1)$ & 899 (21.9) & 0.001 \\
\hline Renal or liver disease & $580(12.3)$ & $503(12.3)$ & 0.95 \\
\hline Chronic obstructive pulmonary disease & $370(7.9)$ & $347(8.5)$ & 0.295 \\
\hline Diabetes & 939 (19.9) & $696(17.0)$ & $<0.001$ \\
\hline Cancer & $596(12.6)$ & $358(8.7)$ & $<0.001$ \\
\hline
\end{tabular}

Bold denotes statistically significant results.

${ }_{n} n(\%)$ unless otherwise indicated.

${ }^{\mathrm{b}}$ Missing data $<6 \%$.

${ }^{\mathrm{c}}$ Those with additional administrative data on comorbidities.

$\mathrm{IQR}$, interquartile range. 
Table 2. Processes of Care Received in Hospital and Discharge Information Among Australian Stroke Clinical Registry Registrants (2010-2013) by Sex

\begin{tabular}{|c|c|c|c|}
\hline & $\begin{array}{c}M e n \\
\mathrm{n}(\%)\end{array}$ & $\begin{array}{c}\text { Women } \\
\mathrm{n}(\%)\end{array}$ & $\mathrm{p}$-Value \\
\hline \multicolumn{4}{|l|}{ Processes of care } \\
\hline Treated in stroke units & $4,103(81.1)$ & $3,434(78.4)$ & 0.258 \\
\hline Intravenous thrombolysis ${ }^{a}$ & $519(12.8)$ & $418(12.1)$ & 0.298 \\
\hline Discharged on antihypertensives ${ }^{\mathrm{b}}$ & $3,133(69.5)$ & $2,568(68.9)$ & 0.634 \\
\hline Care plan for discharge ${ }^{c}$ & $1,138(49.0)$ & $866(47.5)$ & 0.487 \\
\hline \multicolumn{4}{|c|}{ Additional processes of care (Queensland; 21 hospitals) } \\
\hline Number of cases & $1,574(53.0)$ & $1,398(47.0)$ & $<0.001$ \\
\hline Mobilization $^{\mathrm{d}}$ & $515(59.1)$ & $454(52.1)$ & 0.057 \\
\hline Mobilization $\leq 48$ hours $^{\mathrm{d}}$ & $383(44.0)$ & $351(40.3)$ & 0.764 \\
\hline Swallow assessment $\leq 24$ hours & $762(48.4)$ & $604(43.2)$ & 0.389 \\
\hline Aspirin administration $\leq 48$ hours $^{\mathrm{a}}$ & $788(60.2)$ & $570(49.6)$ & 0.042 \\
\hline Antiplatelets/antithrombotics ${ }^{\mathrm{a}, \mathrm{b}}$ & $755(83.0)$ & $579(79.6)$ & 0.666 \\
\hline \multicolumn{4}{|l|}{ Discharge information } \\
\hline Length of stay, median (IQR) days ${ }^{\mathrm{b}}$ & $6(3-10)$ & $6(3-12)$ & 1.000 \\
\hline \multicolumn{4}{|l|}{ Discharge destination } \\
\hline Home & $2,150(42.5)$ & $1,486(33.9)$ & $<0.001$ \\
\hline Inpatient rehabilitation & 1,510 (29.9) & 1,339 (30.6) & \\
\hline Residential aged care facility & $174(3.4)$ & $337(7.7)$ & \\
\hline Other hospitals/facilities & $764(15.1)$ & $668(15.2)$ & \\
\hline Died in hospital & $461(9.1)$ & $552(12.6)$ & \\
\hline
\end{tabular}

Missing data on evidence based therapy were assumed to be "unreceived"; denominators for all registrants unless otherwise indicated. ${ }^{\mathrm{a}}$ Only applies to patients with ischemic strokes.

${ }^{\mathrm{b}}$ Only applies to patients who were discharged.

${ }^{\mathrm{c}}$ Only applies to patients discharged to community (e.g., to a home setting or institutional residential aged care).

${ }^{\mathrm{d}}$ Only applies to patients unable to walk on admission.

For other CVD mortality at 1 year after stroke, women more often died from atrial fibrillation $(49.2 \%$ vs. men $45.0 \%)$ and heart failure $(5.5 \%$ vs. men $3.1 \%)$ than men. Among those who died due to cancer, more men than women died from urinary tract cancer (men $7.5 \%$ vs. $2.9 \%$ ) or skin cancer (men 9.2 vs. $5.7 \%$ ), while more women than men died from cancer of the lung $(18.6 \%$ vs. men $11.7 \%)$ or digestive system (32.9\% vs. men 25.8\%; Supplementary Table S2).

\section{Factors contributing to sex difference in mortality}

All causes. Data were available for 94\% $(n=8,889)$ of the 9,411 patients because of missing data on covariates, including age and stroke severity. Women were 39\% more likely to die by 1 year after stroke than men in the unadjusted

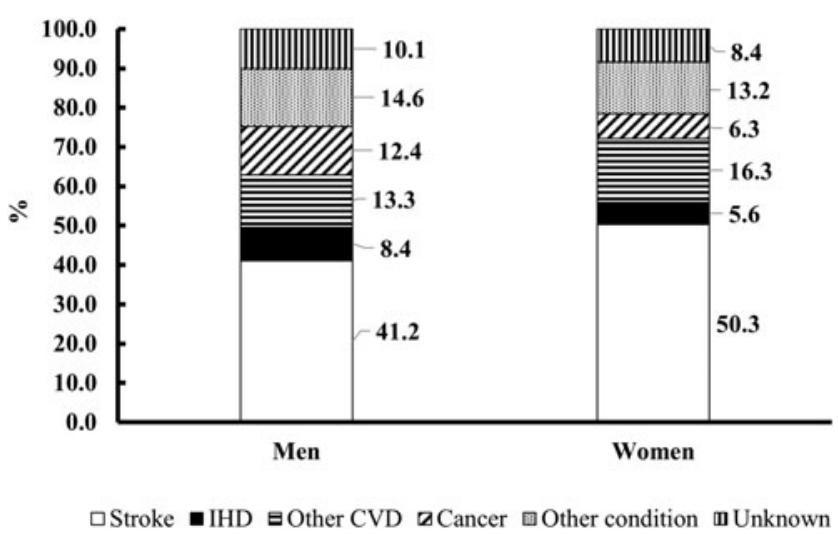

FIG. 1. Causes of death up to 1 year after stroke by sex. IHD, ischemic heart disease; CVD, cardiovascular disease. analysis (women:men hazard ratio [HR] 1.39, 95\% CI 1.23 1.56). Advanced age and more severe strokes in women, compared to men, explained their greater all-cause mortality

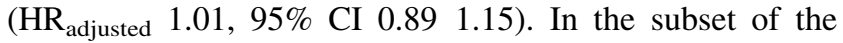
Queensland patients, women also had greater all-cause mortality ( $\mathrm{HR}_{\text {unadjusted }} 1.30,95 \%$ CI 1.08 1.57). Women's advanced age, more severe strokes, and lower likelihood of early aspirin administration fully accounted for the sex difference $\left(\mathrm{HR}_{\text {fully-adjusted }} 0.84,95 \%\right.$ CI 0.691 .01 ; aspirin explained $\sim 16 \%$ of the difference).

Cause-specific (Fig. 2) stroke related. Women had a $65 \%$ greater risk of death associated with stroke than men ( $\mathrm{sHR}_{\text {unadjusted }} 1.65,95 \%$ CI 1.42 1.91; Table 3). The sex difference was greatly attenuated after adjustment for age and stroke severity ( $\mathrm{sHR}_{\text {adjusted }} 1.19,95 \%$ CI 1.02 1.40; Table 3 ). In the subset of Queensland patients, women had greater stroke-specific mortality $\left(\mathrm{sHR}_{\text {unadjusted }} 1.61,95 \%\right.$ CI 1.11 2.34) than men. Women's advanced age, more severe strokes, and lower likelihood of early aspirin administration fully accounted for the sex difference $\left(\mathrm{sHR}_{\text {fully-adjusted }} 0.95,95 \%\right.$ CI 0.66 1.34; aspirin explained $\sim 13 \%$ of the difference).

Ischemic heart disease. Women appeared to have a lesser risk of death due to IHD ( $\mathrm{sHR}_{\text {unadjusted }} 0.88,95 \% \mathrm{CI}$ 0.651 .18 and $\mathrm{sHR}_{\text {adjusted }} 0.58,95 \%$ CI 0.430 .77 ), but they had more deaths from other CVD, that is, atrial fibrillation and heart failure $\left(\mathrm{sHR}_{\text {unadjusted }} 1.65,95 \%\right.$ CI 1.292 .12 ; Table 3). The greater risk of death due to other CVD in women was similarly accounted for by age and stroke severity (sHR adjusted $_{1.12,95 \%}$ CI 0.851 .48 ; Table 3$)$. 


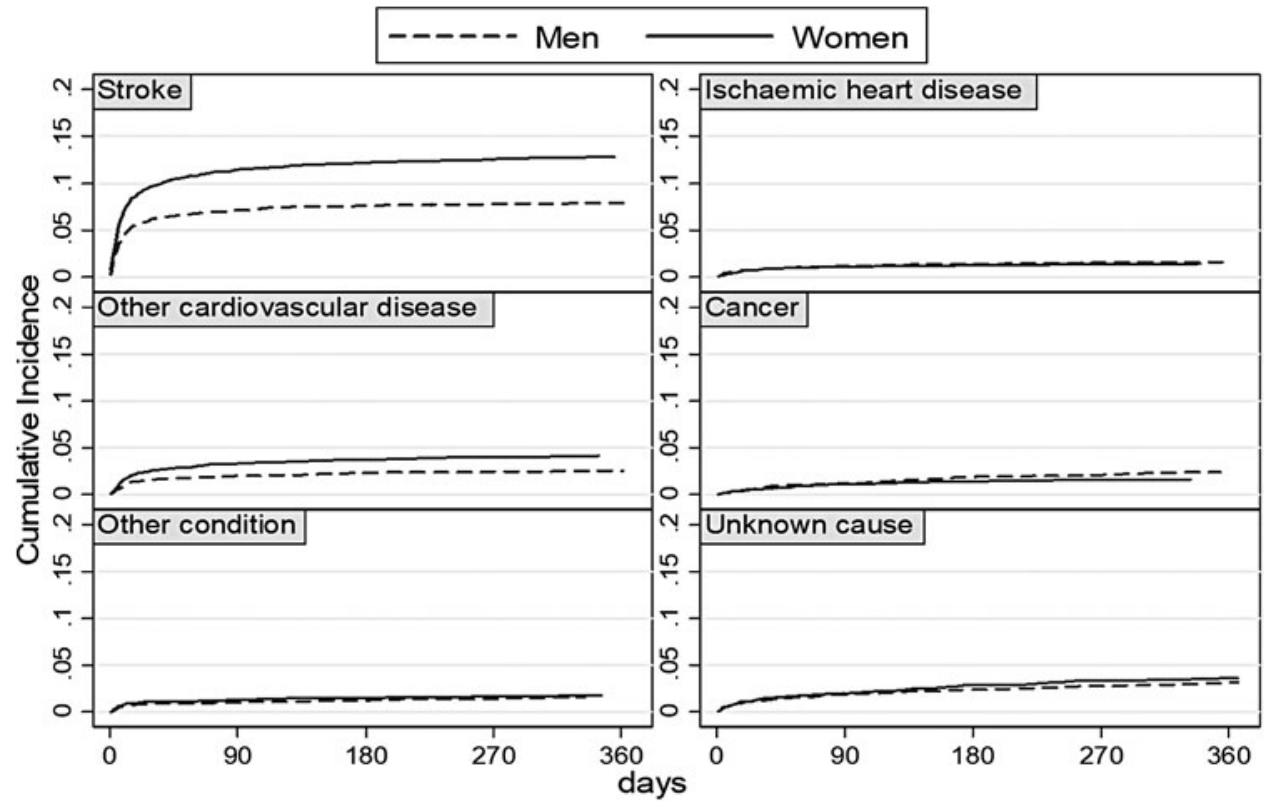

FIG. 2. Cumulative incidence of cause-specific death up to 1 year after stroke.

Cancer. The risk of death attributable to cancer was less in women ( $\left.\mathrm{sHR}_{\text {unadjusted }} 0.69,95 \% \mathrm{CI} 0.500 .95\right)$ than in men and the sex difference remained significant after accounting for age (Table 3). None of the factors, including stroke severity (indicated by being unable to walk independently on admission; Supplementary Table S3), stroke type (Supplementary Fig. S3), time to death (Supplementary Table S4), indicators of processes of care available among all hospitals, and other covariates (e.g., risk factors or comorbidities), confounded or modified the sex difference in cause-specific mortality after stroke.

\section{Sex difference in excess death rates after stroke}

Compared to the general Australian population, patients with a first-ever stroke surviving to 30 days had more than an eight-fold increased risk of death (standardized for age) due to the index stroke or a recurrent stroke within 1 year after stroke, irrespective of sex (SMR, Women: 8.8; Men: 8.3; $p=0.656$ ). The absolute excess risk for stroke was 35.0/1,000 person-years in women and 17.4/1,000 person-years for men
(Table $4 ; p<0.001$ ). People with stroke were also more likely to die from IHD within 1 year than the general population, but the excess mortality, standardized for age, was only slightly greater in men (SMR men 3.5 vs. 3.0; $p=0.310$ ). Conversely, the excess mortality risk for other CVDs was greater in women than men (SMR 3.6 vs. 2.8; $p=0.026$ ) compared to the general population. Men and women with stroke shared an equal 2.6 times greater risk of death from cancer, although the numbers were small (Table 4).

\section{Discussion}

In this study, we found that mortality up to 1 year after stroke was mostly associated with CVDs and cancer, and the specific-cause mortality differed by sex. Women had more deaths due to stroke and other CVDs (not including IHD), while men had greater risks of death from IHD or cancer. Women's advanced age and more severe strokes contributed to the increased risk of death due to stroke and other CVD (i.e., atrial fibrillation and heart failure). In a subset of the cohort, women with ischemic stroke were less often treated

Table 3. Specific Hazard Ratio of Death Up to One Year After Stroke for Women Compared to Men in Australian Stroke Clinical Registry 20102013 Using Competing Risk Models

\begin{tabular}{|c|c|c|c|c|}
\hline \multirow[b]{2}{*}{ Cause of death } & \multirow[b]{2}{*}{ sHR $(95 \%$ CI); unadjusted } & \multicolumn{3}{|c|}{ sHR $(95 \%$ CI $) ;$ adjusted for } \\
\hline & & Age & Stroke severity ${ }^{\mathrm{a}}$ & Age and severity \\
\hline Stroke & $1.65(1.421 .91)^{b}$ & $1.28(1.101 .49)^{\mathrm{b}}$ & $1.46(1.251 .71)^{\mathrm{b}}$ & $1.19(1.021 .40)^{\mathrm{b}}$ \\
\hline Ischemic heart disease & $0.88(0.651 .18)$ & $0.60\left(\begin{array}{lll}0.45 & 0.79\end{array}\right)^{\mathrm{b}}$ & $0.82(0.601 .12)$ & $0.58\left(\begin{array}{lll}0.43 & 0.77)^{\mathrm{b}}\end{array}\right.$ \\
\hline Other CVD & $1.65(1.292 .12)^{\mathrm{b}}$ & $1.19(0.911 .56)$ & $1.49(1.161 .93)^{\mathrm{b}}$ & $1.12(0.851 .48)$ \\
\hline Cancer & $0.69(0.500 .95)^{\mathrm{b}}$ & $0.67(0.500 .91)^{\mathrm{b}}$ & $0.69\left(\begin{array}{lll}0.50 & 0.94)^{\mathrm{b}}\end{array}\right.$ & $0.67(0.490 .91)^{\mathrm{b}}$ \\
\hline Other conditions & $1.17\left(\begin{array}{ll}0.87 & 0.58\end{array}\right)$ & $0.99(0.701 .36)$ & $1.08(0.791 .47)$ & $0.94(0.681 .30)$ \\
\hline Unknown & $1.12(0.841 .49)$ & $0.89(0.641 .21)$ & $1.05(0.781 .40)$ & $0.86(0.631 .17)$ \\
\hline All-cause & $1.39(1.231 .57)^{\mathrm{b}}$ & $1.08(0.951 .22)^{\mathrm{b}}$ & $1.26(1.111 .44)^{\mathrm{b}}$ & $1.01(0.891 .16)$ \\
\hline
\end{tabular}

\footnotetext{
${ }^{a}$ Walking independently on admission was used as a proxy for stroke severity (unable to walk=more severe and able to walk $=$ less severe).

${ }^{\mathrm{b}}$ Statistically significant results.

sHR, specific hazard ratio; CI, confidence interval; CVD, cardiovascular disease.
} 


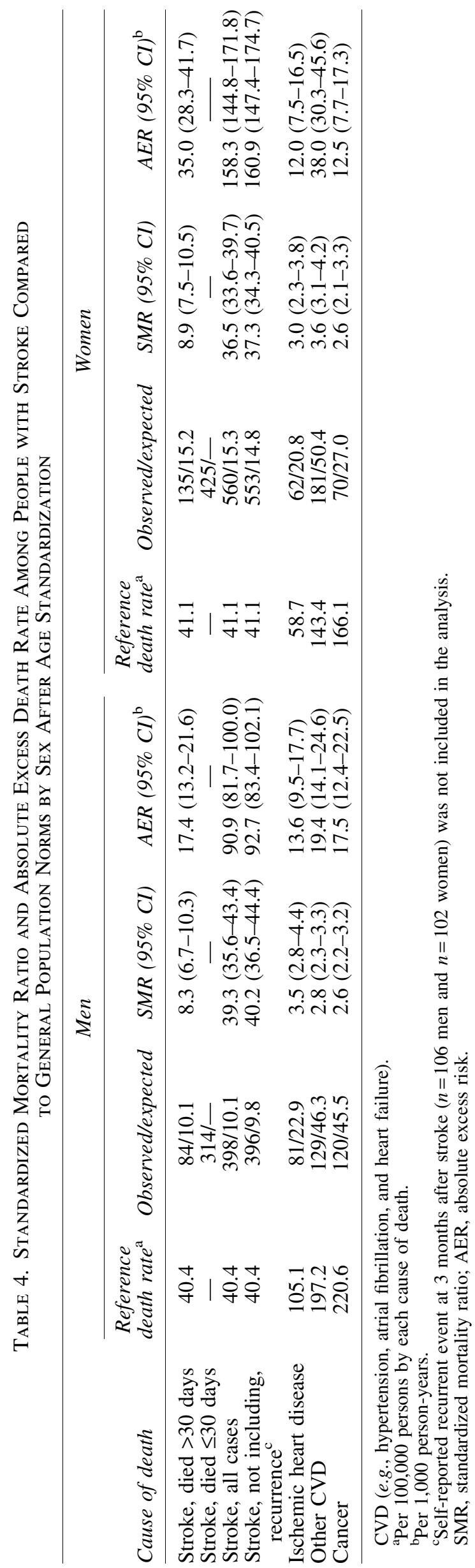


with aspirin $\leq 48$ hours compared to men and this explained part of the sex difference in stroke-specific mortality. None of the other evidence-based therapies contributed to the sex differences in specific-cause mortality after stroke. However, evidence of undertreatment for both sexes was noted for secondary prevention strategies and the provision of a discharge care plan.

In the first year following stroke, both men and women surviving to 30 days had at least an eightfold excess mortality from stroke compared to people of the same age and sex in the general population. Furthermore, they were 3 times more likely to die from other CVD and 2.6 times more likely to die from cancer than the general population, consistent the research by Bronnum-Hansen et al. conducted 15 years ago. ${ }^{6}$

\section{More deaths due to stroke and other CVD in women}

Age was the most important factor contributing to $51 \%$ of the sex differences in stroke-specific mortality. Older age can reflect the multimorbidity ${ }^{15}$ and underreceipt of evidencebased stroke care,${ }^{20}$ both of which are associated with poorer survival after stroke. It is critical to develop clinical recommendations that incorporate age-related complexities (e.g., multimorbidities and polypharmacy) as these may optimize the outcomes of older patients with stroke.

In our study, stroke severity accounted for $24 \%$ of the greater stroke mortality in women than men, and is supported by the evidence from older studies. ${ }^{1}$ In our earlier work using data from eight population-based studies, ${ }^{21}$ more severe strokes in women compared to men were partly explained by age, prestroke dependency, and atrial fibrillation. Strategies to improve prestroke health in the elderly, the majority of whom are women, including targeting modifiable factors contributing to stroke severity (e.g., atrial fibrillation ${ }^{22}$ ), could lessen these sex differences. Further research on the potential biological origin of sex differences in stroke severity is needed to better understand these disparities. The ability to walk on admission as a proxy for stroke severity ${ }^{23}$ appeared to be a reliable predictor of mortality outcome in the study as shown by others in stroke. ${ }^{23}$ The collection of NIHSS data in AuSCR was introduced in 2015, and in the future may allow more opportunities to more fully explore the factors underpinning this observation.

The receipt of aspirin within 48 hours of stroke contributed to the sex difference in stroke mortality, but the data were only available in a subset of patients from Queensland hospitals. The undertreatment with antiplatelet or anticoagulants in women has been observed previously, ${ }^{24}$ suggesting a need to ensure greater access to early treatment with aspirin for women. Although the sex difference in aspirin administration was not fully explained by the covariates collected in the AuSCR, other unmeasured factors such as complications and contraindications to treatment (e.g., allergy, bleeding disorder, or uncontrolled hypertension ${ }^{25}$ may play a role. Since mid-2016, we have expanded data collection in the registry to include additional processes of care measures (i.e., aspirin administration) and contraindications. In the future, this may enable more detailed assessment of a potential sex bias in the early treatment, and thus may provide more opportunities to address the sex differences in mortality after stroke.

There was a residual sex difference in stroke-specific mortality after accounting for age and stroke severity. This could be due to other unmeasured factors such as preexisting functional limitations and presence of atrial fibrillation that were found to be contributors to the greater mortality in women. ${ }^{1}$

The greater risk of death due to other CVD (excluding IHD) in women, particularly atrial fibrillation (AF) and heart failure, was consistent with the sex differences in the comorbidity profile of those with stroke; women were older and frailer, and more often had AF and hypertension than men. ${ }^{1}$ Suboptimal management of AF such as undertreatment with anticoagulants among older patients, ${ }^{26}$ many of whom are women, underpins the need for better detection and treatment of $\mathrm{AF}$ in older men and women.

\section{Fewer deaths due to IHD in women}

Men develop IHD and stroke at a younger age, and have less severe stroke, than women, this being reflected in the greater risk of death from IHD in men. Men with stroke also present with poorer lifestyle risk factors (e.g., smoking and alcohol consumption) or medical histories (i.e., more often having a history of coronary heart disease) that may lead to their higher mortality from IHD. ${ }^{27}$

\section{The role of stroke severity in mortality due to CVD}

Stroke severity ${ }^{23}$ was associated with increased risk of death due to IHD and other CVD, and also contributed to greater cause-specific mortality in women. It may, however, also be affected by prestroke factors, which concurrently affect mortality such as frailty. ${ }^{28}$ Although uncertainty exists over the causal pathway of the association between stroke severity and increased risk of dying from CVD, there are some potential explanations. Inability to walk on admission could reflect the presence of functional limitation before stroke that is associated with chronic diseases, including cardiovascular conditions and frailty. ${ }^{28}$ Although early mobilization is recommended in clinical guidelines ${ }^{29,30}$ to improve walking and functional independence, ${ }^{31}$ only $40 \%$ of patients were mobilized $\leq 48$ hours of admission in our study. This suggests that restricted mobility caused by stroke (i.e., hemiplegia and paraplegia), indicated by walking ability on admission, may persist into the longer term following stroke. Those people with restricted mobility (preexisting or following stroke) face a high risk of venous thromboembolism and cardiovascular events, ${ }^{32}$ and subsequent development of frailty, which is also associated with increased mortality in CVD, including stroke. ${ }^{33}$

\section{Excess deaths due to stroke, CVD, and cancer for both sex}

Men and women with stroke had notable excess deaths due to stroke, other CVD, and cancer when compared to the general population, even after age standardization. These diseases share common risk factors, such as hyperlipidemia, diabetes, and smoking, ${ }^{34}$ and potentially relate to a common mechanism (atherosclerosis). ${ }^{35}$ Primary prevention strategies should be prioritized by targeting vascular risk factors and comorbidities to improve survival following stroke. In addition, a large number of men and women with stroke were not treated with evidence-based therapies, including management in a stroke unit, intravenous thrombolysis for 
ischemic stroke, and secondary prevention medication at the time of discharge (Table 2). Only $70 \%$ of AuSCR registrants were prescribed antihypertensive medications at discharge from hospital. This is concerning as we have previously shown that patients not prescribed these agents experienced worse long-term survival outcomes than those prescribed these agents before discharge from hospital. ${ }^{36}$ Therefore, we should continuously strive to improve access to evidencebased care in Australia after acute stroke, regardless of gender, to promote a better chance of survival. ${ }^{9}$ One way to increase the use of evidence-based care is through quality improvement programs. ${ }^{37-40}$ If hospitals provided care in line with evidence-based clinical guidelines, the health and economic benefits of reducing the impacts of stroke would be substantial within the Australian context. ${ }^{41}$ Moreover, because patients with stroke and a history of cancer have an increased risk of recurrent stroke and cardiovascular mortality, $^{42}$ further examination on predictors of long-term outcomes among people with stroke and cancer is needed to inform future interventions.

\section{Strengths and limitations}

To our knowledge, this is the first time a national stroke registry has been used to examine sex differences in causespecific mortality 1 year after stroke. Our research also provides the excess death rate according to each cause of death (i.e., stroke, IHD, other CVD, and cancer) for both men and women compared to the Australian general population. The findings were based on a large high-quality dataset, enabling us to have enough reliability and power to test our hypotheses. Stroke performance indicators in the AuSCR were aligned with high-priority national clinical recommendations, making our work relevant to current clinical practice. The AuSCR data on survival and cause of death linked to the national registration minimized selection bias from missing death records (only $10 \%$ of registrants had unknown cause of death). We were able to assess the roles of a wide range of covariates in sex differences in cause of death, particularly risk factors for stroke and comorbidities through linked administrative data.

We acknowledge some limitations of the study. Some potential confounding factors of the sex differences in mortality after stroke, including living arrangement before stroke, prestroke function, withdrawal of care during the hospitalization, and other processes of care (e.g., being discharged on statins), were unavailable. In our earlier work using data from 13 population-based studies, ${ }^{1}$ we found preexisting functional limitations, but not differences in stroke care, contributed to the greater mortality in women. The AuSCR sample was consistent with other representative stroke populations. 9 For example, the all-cause mortality by 1 year (22\%) was comparable to other similar studies worldwide. ${ }^{1}$ However, patients registered in the AuSCR hospitals during the study period were mainly admitted to neurology/stroke units and from metropolitan areas. ${ }^{9}$ The possibility of bias from these attributes cannot be eliminated, which is another limitation in relationship to generalizability of the findings. According to a recent study of the accuracy of the Australian NDI, attributing deaths to CVD (cardiac diseases and stroke) was more likely to be inaccurately recorded compared to cancer-related deaths. ${ }^{43}$ However, sex differ- ences were unlikely to be affected because similar inaccuracy of the national registration is expected for both groups.

\section{Conclusions}

We found that women, compared to men, more often died at 1 year after stroke and the cause-specific mortality varied by sex. Women had more deaths attributed to stroke or other CVD than men, and the difference was mostly accounted for by their advanced age and stroke severity, rather than other factors. Compared with the general population, excess mortality for both sexes was mostly attributed to stroke or recurrent events, other cardiovascular conditions, and cancer. Our findings reinforce the need for better primary and secondary prevention of stroke and CVD for men and women by targeting cardiovascular risk factors and comorbidities. Suboptimal prescribing of prevention medications at discharge among both sexes highlights the importance of improving access to evidence-based care to all people who suffer stroke as this is associated with better survival and other outcomes.

\section{Data Availability Statement}

The data, analytic methods, and study materials will not be made available to other researchers for purposes of reproducing the results or replicating the procedure. Qualified investigators can request access to patient-level data, analytic methods, and study materials after ethics clearance and approval by all authors.

\section{Acknowledgments}

We acknowledge Joyce Lim, Sabrina Small, Francis Kung, Karen Moss, Steven Street, and Renee Stojanovic from Australian Stroke Clinical Registry (AuSCR) Office who contributed to AuSCR operations during this study period. Staff from The George Institute, the Florey, and the Stroke Foundation are acknowledged for their contributions to patient follow-up. We would also like to thank the hospital staff for their diligence regarding data collection for AuSCR. Hospital site investigators and data collectors between 2010 and 2013 and members of the management and steering committees are also acknowledged (see Supplementary Data S2).

\section{Author Disclosure Statement}

Dr. H.T.P., Dr. S.G., Dr. C.L.B., Dr. A.G.T., Dr. J.K., and Dr. H.C.C. report no disclosures. Dr. D.A.C., Dr. N.A.L., Dr. R.G., Dr. M.F.K., and Dr. C.S.A. are members of the management committee for AuSCR. Dr. D.A.C. has received educational grant for AuSCR from Boehringer Ingelheim paid to her institution. Dr. C.S.A. has received grants and speaker fees from Takeda unrelated to this work. No competing financial interests exist.

\section{Funding Information}

During the study period, Australian Stroke Clinical Registry was supported by grants from the National Health and Medical Research Council (1034415), Allergan, Ipsen, Boehringer Ingelheim, Monash University, Queensland Health, the Stroke Society of Australasia, the Stroke Foundation, and consumer donations. Dr. H.T.P. was supported by 
a Merle Weaver Postgraduate Scholarship (University of Tasmania). The following authors received support from a National Heart Foundation of Australia Future Leader Fellowship: Dr. S.G. (100446) and Dr. N.A.L. (102055). The following authors received research fellowship funding from the National Health and Medical Research Council: Dr. D.A.C. (cofunded Heart Foundation: 1063761), Dr. A.G.T. (1042600), Dr. M.F.K. (1109426), and Dr. C.S.A. (1081356).

\section{Supplementary Material}

Supplementary Data S1 Supplementary Data S2 Supplementary Figure S1 Supplementary Figure S2 Supplementary Figure S3 Supplementary Table S1 Supplementary Table S2 Supplementary Table S3 Supplementary Table S4

\section{References}

1. Phan HT, Blizzard CL, Reeves MJ, et al. Sex differences in long term mortality after stroke in the INSTRUCT (INter national STRoke oUtComes sTudy). A meta analysis of individual participant data. Circ Cardiovasc Qual Outcomes 2017;10:e003436.

2. Phan HT, Gall SL, Blizzard CL, et al. Sex differences in care and long term mortality after stroke: Australian Stroke Clinical Registry. J Womens Health 2019;28:712 720.

3. Ganesh A, Luengo Fernandez R, Wharton RM, et al. Time course of evolution of disability and cause specific mor tality after ischemic stroke: Implications for trial design. J Am Heart Assoc 2017;6:e005788.

4. Howard G, Evans GW, Murros KE, Toole JF, Lefkowitz D, Truscott BL. Cause specific mortality following cerebral infarction. J Clin Epidemiol 1989;42:45 51.

5. Mogensen UB, Olsen TS, Andersen KK, Gerds TA. Cause specific mortality after stroke: Relation to age, sex, stroke severity, and risk factors in a 10 year follow up study. J Stroke Cerebrovasc Dis 2013;22:e59 e65.

6. Bronnum Hansen H, Davidsen M, Thorvaldsen P. Long term survival and causes of death after stroke. Stroke 2001; 32:2131 2136.

7. Reeves MJ, Bushnell CD, Howard G, et al. Sex differences in stroke: Epidemiology, clinical presentation, medical care, and outcomes. Lancet Neurol 2008;7:915 926.

8. Gall SL, Donnan G, Dewey HM, et al. Sex differences in presentation, severity, and management of stroke in a population based study. Neurology 2010;74:975 981.

9. Cadilhac DA, Andrew NE, Lannin NA, et al. Quality of acute care and long term quality of life and survival: The Australian Stroke Clinical Registry. Stroke 2017;48:1026 1032.

10. Cadilhac D, Lannin N, Anderson C, et al. The Australian Stroke Clinical Registry annual report 2013. Heidelberg, Germany: The Florey Institute of Neuroscience and Mental Health, 2015.

11. Australian Institute of Health and Welfare. About national death index. AIHW, 2018. Available at: https://aihw.gov .au/about our data/our data collections/national death index/ about national death index Accessed May 25, 2018.
12. Adams HP, Bendixen BH, Kappelle LJ, et al. Classification of subtype of acute ischemic stroke. Definitions for use in a multicenter clinical trial. TOAST. Trial of Org 10172 in Acute Stroke Treatment. Stroke 1993;24:35.

13. Kilkenny MF, Kim J, Andrew NE, et al. Maximising data value and avoiding data waste: A validation study in stroke research. Med J Aust 2019;210:27 31.

14. Quan H, Li B, Couris CM, et al. Updating and validating the Charlson comorbidity index and score for risk adjust ment in hospital discharge abstracts using data from 6 countries. Am J Epidemiol 2011;173:676 682.

15. Counsell C, Dennis M, McDowall M, Warlow C. Predict ing outcome after acute and subacute stroke: Development and validation of new prognostic models. Stroke 2002;33: 10411047.

16. Greenland S. Modeling and variable selection in epidemi ologic analysis. Am J Public Health 1989;79:340 349.

17. Nichols M, Peterson K, Herbert J, Alston L, Allender S. Australian heart disease statistics 2015. Melbourne, Aus tralia: National Heart Foundation of Australia, 2016.

18. Australian Institute of Health and Welfare. Cancer survival and prevalence in Australia: period estimates from 1982 to 2010. AIHW, 2012. Available at: https://aihw.gov.au/ reports/cancer/cancer survival and prevalence in australia perio/data Accessed December 1, 2017.

19. StataCorp. Stata statistical software: Release 12. College Station, Texas: StataCorp. 2011.

20. Luker JA, Wall K, Bernhardt J, Edwards I, Grimmer Somers KA. Patients' age as a determinant of care received following acute stroke: A systematic review. BMC Health Serv Res 2011;11:161.

21. Phan HT, Reeves MJ, Blizzard CL, et al. Sex differences in severity of stroke in the INSTRUCT study: A meta analysis of individual participant Data. J Am Heart Assoc 2019;8:e010235.

22. Kakar P, Boos CJ, Lip GYH. Management of atrial fibril lation. Vasc Health Risk Manag 2007;3:109 116.

23. Sim J, Teece L, Dennis MS, Roffe C. Validation and re calibration of two multivariable prognostic models for survival and independence in acute stroke. PLoS One 2016; 11:e0153527.

24. Simpson CR, Wilson C, Hannaford PC, Williams D. Evi dence for age and sex differences in the secondary pre vention of stroke in Scottish primary care. Stroke 2005;36: 17711775.

25. Shahpouri MM, Mousavi S, Khorvash F, Mousavi SM, Hoseini T. Anticoagulant therapy for ischemic stroke: A review of literature. J Res Med Sci 2012;17:396 401.

26. Yiin GS, Howard DP, Paul NL, et al. Age specific inci dence, outcome, cost, and projected future burden of atrial fibrillation related embolic vascular events: A population based study. Circulation 2014;130:1236 1244.

27. Bots SH, Peters SAE, Woodward M. Sex differences in coronary heart disease and stroke mortality: A global as sessment of the effect of ageing between 1980 and 2010. BMJ Glob Health 2017;2:e000298.

28. Hubbard RE, Peel NM, Samanta M, Gray LC, Mitnitski A, Rockwood K. Frailty status at admission to hospital pre dicts multiple adverse outcomes. Age Ageing 2017;46: 801806.

29. Powers WJ, Rabinstein AA, Ackerson T, et al. 2018 Guidelines for the early management of patients with acute Ischemic stroke: A guideline for healthcare professionals from the American Heart Association/American Stroke Association. Stroke 2018;49:e46 e110. 
30. Boddice G, Brauer S, Gustafsson L, Kenardy J, Hoffmann T. Clinical guidelines for stroke management. Melbourne, Australia: Stroke Foundation. 2010.

31. Cumming TB, Thrift AG, Collier JM, et al. Very early mobilization after stroke fast tracks return to walking. Stroke 2011;42:153.

32. Kappelle LJ. Preventing deep vein thrombosis after stroke: Strategies and recommendations. Curr Treat Options Neu rol 2011;13:629 635 .

33. Afilalo J, Karunananthan S, Eisenberg MJ, Alexander KP, Bergman H. Role of frailty in patients with cardiovascular disease. Am J Cardiol 2009;103:1616 1621.

34. Koene RJ, Prizment AE, Blaes A, Konety SH. Shared risk factors in cardiovascular disease and cancer. Circulation 2016;133:1104 1114.

35. Soler EP, Ruiz VC. Epidemiology and risk factors of ce rebral ischemia and ischemic heart diseases: Similarities and differences. Curr Cardiol Rev 2010;6:138 149.

36. Andrew NE, Kim J, Thrift AG, et al. Prescription of anti hypertensive medication at discharge influences survival following stroke. Neurology 2018;90:e745 e753.

37. Cadilhac DA, Andrew NE, Kilkenny MF, et al. Improving quality and outcomes of stroke care in hospitals: Protocol and statistical analysis plan for the Stroke123 im plementation study. Int J Stroke 2018;13:96 106.

38. Cadilhac DA, Andrew NE, Stroil Salama E, et al. Improving discharge care: The potential of a new organisational inter vention to improve discharge after hospitalisation for acute stroke, a controlled before after pilot study. BMJ Open 2017; 7:e016010.

39. Middleton S, Lydtin A, Comerford D, et al. From QASC to QASCIP: Successful Australian translational scale up and spread of a proven intervention in acute stroke using a prospective pre test/post test study design. BMJ Open 2016;6:e011568.

40. Middleton S, McElduff P, Ward J, et al. Implementation of evidence based treatment protocols to manage fever, hy perglycaemia, and swallowing dysfunction in acute stroke (QASC): A cluster randomised controlled trial. Lancet 2011;378:1699 1706.

41. Kim J, Andrew NE, Thrift AG, Bernhardt J, Lindley RI, Cadilhac DA. The potential health and economic impact of improving stroke care standards for Australia. Int J Stroke 2017;12:875 885 .

42. Lau KK, Wong YK, Teo KC, et al. Stroke patients with a past history of cancer are at increased risk of recurrent stroke and cardiovascular mortality. PLoS One 2014;9:e88283.

43. Magliano D, Liew D, Pater H, et al. Accuracy of the Australian National Death Index: Comparison with adju dicated fatal outcomes among Australian participants in the Long term Intervention with Pravastatin in Ischaemic Disease (LIPID) study. Aust N Z J Public Health 2003;27: 649653.

Address correspondence to: Dominique A. Cadilhac, PhD Department of Medicine School of Clinical Sciences at Monash Health Monash University Level 3 Hudson Institute Building 2731 Wright Street, Clayton Melbourne, VIC 3168 Australia

E-mail: dominique.cadilhac@monash.edu 